\title{
Electrochemical determination of lead(II) ions in hot spring water based on a multi-walled carbon nanotube-modified glassy carbon electrode
}

\author{
Xiaobiao $\mathrm{Li}^{1}$, Shuxia Hou ${ }^{1}$, Chengji Xie ${ }^{2}$, Guojing $\mathrm{Fan}^{3, *}$ \\ ${ }^{1}$ Inner Mongolia University of Finance and Economics, Hohhot, Inner Mongolia, 010070, P.R. China \\ ${ }^{2}$ Inner Mongolia Technical College of Construction, Hohhot, Inner Mongolia ,010070, P.R. China \\ ${ }^{3}$ School of History Culture \&Tourism, Ganzhou Normal University, Ganzhou, Jiangxi, 341000, P. R. \\ China \\ *E-mail: guojingfan086@sina.com
}

Received: 16 November 2017 / Accepted: 30 December 2017 / Published: 5 February 2018

The present study reports the fabrication of a carbon nanotube/Nafion composite film (MWNT/Nafion)-modified glassy carbon electrode (GCE) along with its excellent performance in the analysis of $\mathrm{Pb}^{2+}$ (favorable catalytic activity, large surface area, high cation exchange capacity, and strong adsorption ability). The stripping peak current of $\mathrm{Pb}^{2+}$ was greatly increased at the MWNT/Nafion-coated GCE compared with the bare and Nafion-coated GCEs. The optimization of different parameters was also carried out, including optimization of the accumulation time, accumulation potential, supporting electrolyte, and MWNT/Nafion suspension volume. Therefore, our developed electrode can be used in the analysis of $\mathrm{Pb}^{2+}$ in real hot spring water samples.

Keywords: Lead ions; Electrochemical sensor; Carbon nanotube; Hot spring water; Glassy carbon nanotube

\section{INTRODUCTION}

Lead is a substance commonly found in the environment and a potential occupational toxin, with human exposure arising from industrial activity, food, drinking water, soil, and paint sources. Lead is highly persistent in the environment, mainly due to its non-biodegradability. The toxicity of lead could result in irreversible health effects to the central nervous, hepatic, circulatory, cardiovascular, reproductive, and renal systems [1-3]. According to the US EPA, the action level for 
lead in drinking water is $15 \mu \mathrm{g} / \mathrm{L}[4,5]$, while it is $10 \mu \mathrm{g} / \mathrm{L}$ according to the WHO [6] (comparable to the restrictions set by the EU) [4, 7].

Electrochemical stripping analysis based on a preconcentrating step of $\mathrm{Pb}(\mathrm{II})$ ions on a suitable working electrode through reduction to metallic lead has been extensively used in the detection of lead at the trace level. Then, the lead deposit was subjected to a stripping step, with rapid oxidation of the metal; meanwhile, the transition time (in stripping chronopotentiometry) or the current (in stripping voltammetry) was obtained. Initially, a mercury electrode, which is ultra-sensitive in lead analysis, was used for the stripping measurement. Unfortunately, mercury electrodes are disadvantageous due to their toxicity. Therefore, a few environmentally friendly substitutes were developed for the analysis of lead. Among them, bismuth film electrodes have been prominent, but it also requires the formation of a bismuth film on a suitable substrate, which would increase the workflow complexity [8]. Additionally, some problems related to the multiple stripping peaks would arise when using solid electrodes, possibly due to the interactions between co-deposited metals and/or underpotential/bulk deposition of the target metals. Substantial efforts have been exerted to investigate multiple peaks in the simultaneous detection of copper and lead on glassy carbon, graphite and boron-doped diamond electrodes [5, 9-12]. However, these composite materials have advantages such as fast preparation and low cost and have thus been applied to lead analysis [13-16]. The preparation strategy of injectionmolding has been reported in previous studies, in addition to its merits and demerits in the mass production of polymer-based lab-on-a-chip analytical apparatuses [17-19]. Additionally, the feasibility of the plastic/carbon fiber composite electrodes in electrochemical analysis has been confirmed in previous literature [20-24].

Multi-walled carbon nanotubes (MWNTs) consist of concentric cylinders located around a common central hollow with a $0.34-\mathrm{nm}$ spacing between the layers close to that of the interlayer distance in graphite. MWNTs are characterized by distinct optical, electrical, thermal, mechanical, and structural features and have the potential to be used for many different purposes [25-28]. In addition, MWNTs promote the study of their uses as new electrode materials in the field of electrochemistry due to their remarkable catalytic activity and subtle electronic properties [29-37].

In this work, we intended to propose a convenient, sensitive, and selective route to analyze $\mathrm{Pb}^{2+}$ based on the synergistic effects of Nafion and MWNTs. As a perfluorinated sulphonated cation exchanger, Nafion has a high cation exchange capacity, thus having been widely used for electrode modification in many different cases, including enhancing the selectivity and sensitivity of the analysis of positively charged species.

The present study reported the preparation of a homogeneous and stable carbon nanotube/Nafion composite (MWNT/Nafion) suspension through the dispersion of MWNTs in ethanol containing $1 \%$ Nafion. However, the surface of a glassy carbon electrode (GCE) was modified with the MWNT/Nafion composite film. In terms of the final MWNT/Nafion film, MWNTs have many advantages, including high surface area, high catalytic activity, and distinct adsorption ability; and Nafion is highly cationically selective. Thus, the electrochemical response of $\mathrm{Pb}^{2+}$ could be improved at the MWNT/Nafion-coated GCE, leading to a highly selective and sensitive detection in the analysis of $\mathrm{Pb}^{2+}$. 


\section{EXPERIMENTS}

\subsection{Device and chemicals}

An RST3000 electrochemical system (Suzhou Risetech Instrument Co., Ltd., Suzhou, China) was used throughout the voltammetric experiments. A typical three-electrode geometry was employed, where the working, auxiliary, and reference electrodes were a bare GCE or Nafion/MWNTs filmcoated GCE, a platinum wire, and a saturated calomel electrode (SCE). A PHS-3C precision pH meter (Leici Devices Factory of Shanghai, China) was used for the pH test, with calibration performed every day using a standard buffer solution.

MWNTs were provided by Shenzhen Nantotech Port Co., Ltd. Nafion (5\%) was commercially available from Aldrich. $\mathrm{Pb}\left(\mathrm{NO}_{3}\right)_{2}$ (Shanghai Reagent Corporation, China) was dissolved in bi-distilled water for the preparation of a $0.01 \mathrm{M} \mathrm{Pb}^{2+}$ stock solution. The water was bi-distilled. Furthermore, all other reagents were of analytical grade and used without further purification.

\subsection{Preparation of a Nafion/MWNTs composite film-modified GCE}

For the pretreatment of the bare GCE, a $0.05 \mu \mathrm{m}$ alumina slurry on a polishing cloth was first used, followed by thorough rinsing using $\mathrm{HNO}_{3}-\mathrm{H}_{2} \mathrm{O}(1: 1$, v/v), along with washing using pure ethanol and redistilled water, respectively. A $68 \mathrm{wt} \%$ concentrated nitric acid was mixed with the untreated MWNTs $(10 \mathrm{mg}$ ), followed by sonication for approximately $240 \mathrm{~min}$. Then, the mixed solution was filtered and washed using double-distilled water until a litmusless state of the filtrate was reached. The treated MWNTs were left to dry under an infrared lamp. Nafion/MWNTs suspension was prepared by sonicating the treated MWNTs $(5.0 \mathrm{mg}$ ) in Nafion methanol solution (wt. $0.1 \% ; 10.0 \mathrm{ml}$ ) for approximately $0.5 \mathrm{~h}$ until a homogeneous suspension was reached. For comparison, the preparation of the MWNTs suspension followed the same procedure, using N,N-dimethylformamide (DMF) instead of a $0.1 \%$ Nafion solution. After evenly coating the pretreated GCE using the Nafion/MWNTs suspension $(10.0 \mu \mathrm{L})$, methanol was evaporated off at ambient temperature. For comparison, the preparation of the Nafion-modified GCE followed the same procedure, only without MWNTs. After the DMF was evaporated under an ultraviolet lamp, the MWNTs/GCE was yielded. Prior to the test, the coated electrode was repeatedly washed using double-distilled water to remove the loosely combined modifiers. The Nafion/MWNT-coated electrode was stored in a pH 7.0 phosphate solution and could be used for 80 cyclic voltammetric (CV) cycles.

\subsection{Analytical Procedure}

The $\mathrm{Pb}^{2+}$ measurement consists of the following 3 major steps: accumulation, electrochemical reduction, and stripping out. First, the electrode was immersed in a solution of $\mathrm{Pb}\left(\mathrm{NO}_{3}\right)_{2}$ under stirring at $300 \mathrm{rpm}$ for $10 \mathrm{~min}$, to accumulate $\mathrm{Pb}^{2+}$ onto the MWNT-coated GCE (open-circuit potential). This was followed by a complete rinse of the as-prepared MWNTs/GCE/ $/ \mathrm{Pb}^{2+}$ using deionized water, along with drying using a soft tissue. Then, the final electrode was transferred into a separate voltammetric 
cell that contained a $\mathrm{pH} 4.4$ lead-free fresh supporting electrolyte ( $0.2 \mathrm{M}$ acetate buffer). The electrochemical reduction of $\mathrm{Pb}^{2+}$ was carried out after nitrogen bubbling through the as-prepared solution for de-aeration at an applied potential of $-1.1 \mathrm{~V}$. Furthermore, differential pulse anodic stripping voltammetry (DPASV) was carried out for the quantitative analysis of $\mathrm{Pb}^{2+}$. The optimum parameters were as follows: pulse width: $0.01 \mathrm{~s}$; amplitude: $0.05 \mathrm{~V}$; pulse period: $0.2 \mathrm{~s}$; rest time: $15 \mathrm{~s}$; sampling width: $0.005 \mathrm{~s}$. After each experiment, the produced residual lead was removed from the surface of the electrode through the regeneration of the MWNT-coated GCE in a fresh stirred supporting electrolyte by electrolysis for $2 \mathrm{~min}$ at $+0.3 \mathrm{~V}$. A comparative study was performed using electro-accumulated $\mathrm{Pb}^{2+}$ at an applied potential of $-1.1 \mathrm{~V}$ for $10 \mathrm{~min}$.

\subsection{Collection and preparation of hot spring water specimens}

The hot spring water test samples were collected from Aer Mountain, Inner Mongolia using a 50-mL plastic tube. Prior to the test, the samples were filtered through a filter paper (pore size: 200 $\mathrm{nm}$ ). To assess the accuracy of the experimental results, these environmental samples were analyzed with the standard addition method.

\section{RESULTS AND DISCUSSION}

The SEM image of Nafion/MWNT film on the GCE is shown in Fig. 1. The uniform coating of this film on the surface of the electrode was apparently observed along with the generation of a spaghetti-like porous reticular structure. This distinct structure provided a large surface area compared with the apparent geometric area. The connection between MWCNTs could also result in a high conductivity.

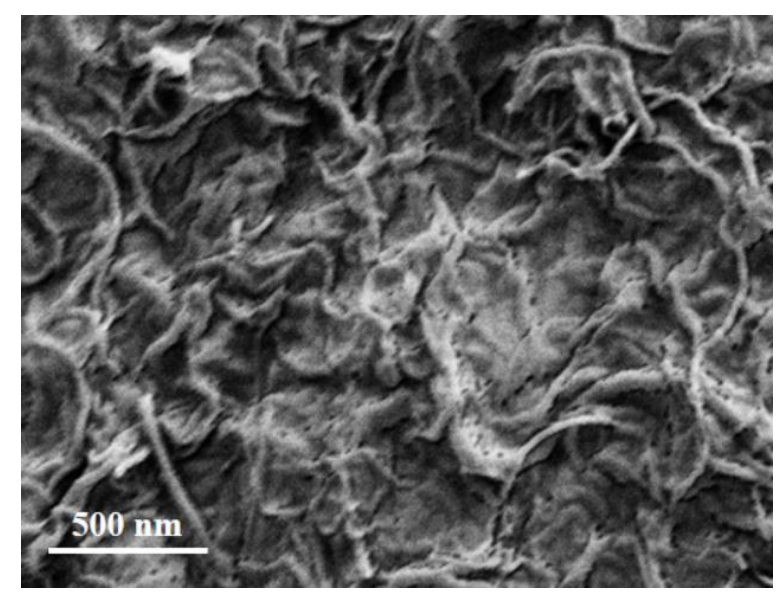

Figure 1. SEM image of Nafion/MWNTs film on GCE. 
Because of its high sensitivity and excellent resolution, differential pulse dissolution voltammetry (DPASV) is a perfect electrochemical method for trace level determination. The accumulation of $\mathrm{Pb}^{2+}$ from the solution phase occurred on the MWNT-coated GCE. The DPASV characterization recorded for the $\mathrm{Pb}^{2+}(0.1 \mu \mathrm{M})$ in $0.1 \mathrm{M}$ of $\mathrm{pH} 5.0$ acetate buffer solution at the bare GCE and the MWNT-coated GCE is shown in Fig. 2, along with that recorded in $0.1 \mathrm{M}$ of pH 5.0 acetate buffer solution at the MWNT-coated GCE. When the potential was applied as $-0.62 \mathrm{~V}$, the MWNT-coated GCE showed a lower stripping peak current than the bare GCE, which is ascribed to the absorption ability of the MWNTs. The stripping voltammetric experiment was carried out with the following procedure. First, $\mathrm{Pb}^{2+}$ was accumulated from the solution phase onto the MWNT/GCE surface through selective complexation with non-bonding electron pairs of oxygen in hydroxy groups of terephthalic acid to yield a metal-ligand complex. This was followed by the reduction of the accumulated complexed ions in the modification layer at a constant voltage of $-1.1 \mathrm{~V}$ applied to the accumulation. Subsequently, a positive scanning potential was applied with a differential pulse voltammetric technique (DPV) to electrochemically strip the lead back into the solution. For the MWNTs, the diffusion of $\mathrm{Pb}^{2+}$ into the bulk structure resulted from their highly porous and flexible channels; meanwhile the $\mathrm{Pb}^{2+}$ could be selectively adsorbed due to their pore size and shape. Therefore, the combined effect resulted in an increasing amount of $\mathrm{Pb}^{2+}$ accumulated on the MWNT/GCE surface [38, 39]. Additionally, MWNTs were highly permeable in water and have a distinct film-generation capacity. Immobilization agents were not needed, and thus, neither the electron transfer across the film nor the electrode conductivity would suffer. Moreover, Nafion is a cation exchanger with a high cation exchange capacity. Therefore, the Nafion film-modified GCE shows a higher accumulation efficiency for $\mathrm{Pb}^{2+}$ and finally enhances the stripping current $[1,40,41]$.

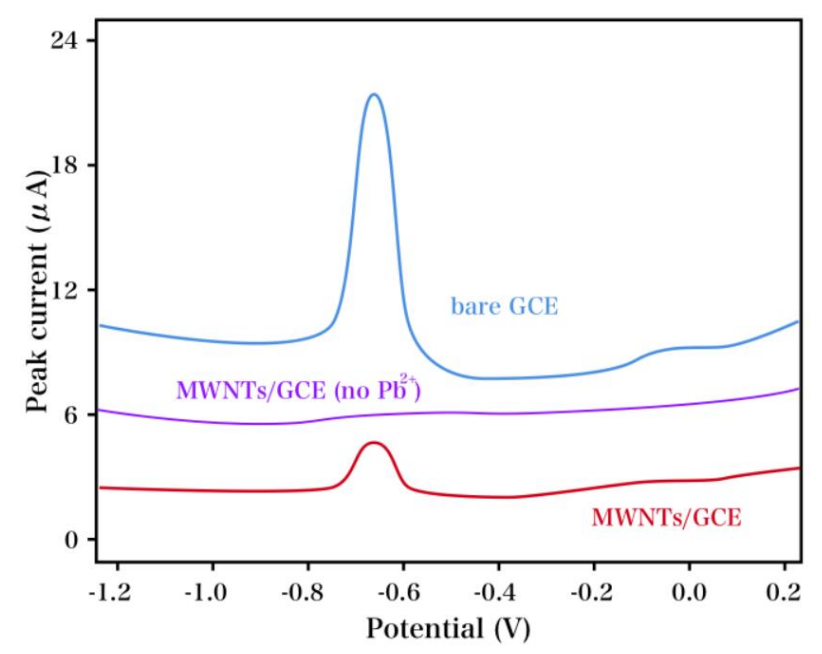

Figure 2. DPASV curve recorded for $\mathrm{Pb}^{2+}(0.1 \mu \mathrm{M})$ in a $0.1 \mathrm{M}$ acetate buffer solution of $\mathrm{pH} 5.0$ at the bare GCE and the MWNT-coated GCE; and DPASV curve recorded in a $0.1 \mathrm{M}$ acetate buffer solution of $\mathrm{pH} 5.0$ at the MWNT-coated GCE. 
Measurements were taken using an optimized system based on that developed by Dai et al. [42], with $0.1 \mathrm{M} \mathrm{HCl}$ as the supporting electrolyte. Fig. 3A shows the $\mathrm{pH}$ influence on the DPASV response over a range of 3.5 to 5.5. With the $\mathrm{pH}$ increase in a range of 3.5 to 5.0, an apparent increase in the peak current was found, reaching the maximal value at $\mathrm{pH}$ 5.0. In addition, as the $\mathrm{pH}$ was increased further to 5.5, a decrease in peak current was observed, possibly due to the lower response at low $\mathrm{pH}$ caused by the electrostatic repulsion between $\mathrm{Pb}^{2+}$ and protonated hydroxy groups of the MWNTs. The current response of $\mathrm{Pb}^{2+}$ was increased by the electrostatic attraction, where the number of protonated hydroxy groups was reduced by the increase in $\mathrm{pH}$. In more basic media, the peak current for the lead stripping peaks was found to diminish in magnitude. Conversely, in more acidic solutions, the lead stripping peaks became increasingly sharp. These changes are attributed to the formation of lead hydroxide in the more basic media. Each lead hydroxide species has a different reoxidation half-wave potential causing multiple or broadened stripping peaks [43]. On the other hand, as the $\mathrm{pH}$ value increase, an increase in the hydrolysis degree of the metal ions was found. In addition, at higher $\mathrm{pH}$, a white $\mathrm{Pb}(\mathrm{OH})_{2}$ precipitate was possibly generated. These results showed that the $\mathrm{pH}$ was optimized as 5.0, and used for the following experiments.

A

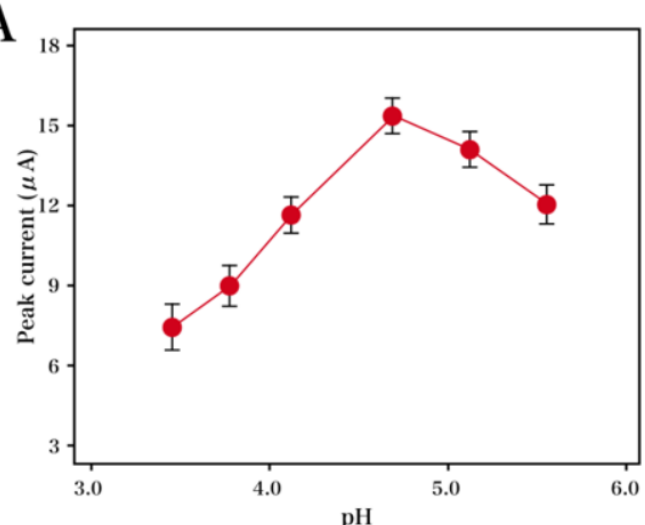

$\mathrm{C}$

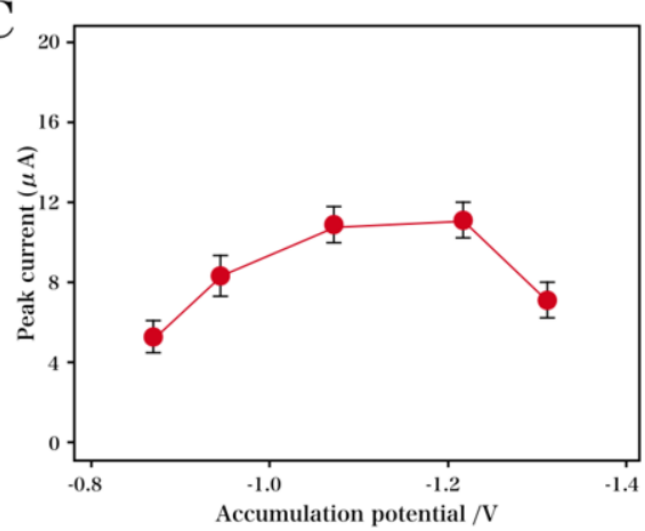

B

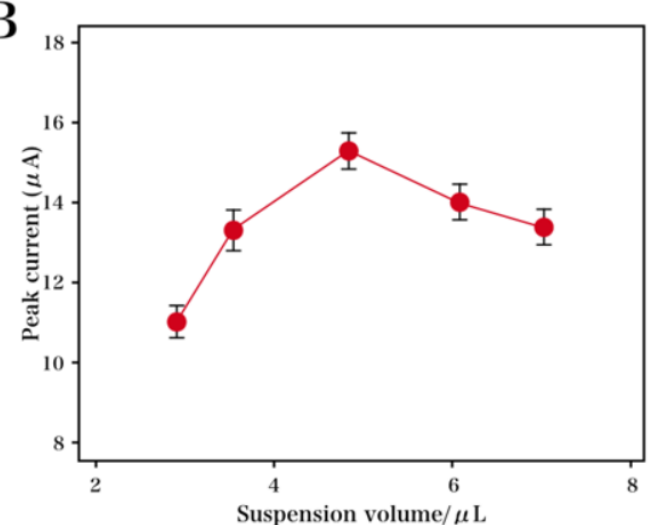

$\mathrm{D}$

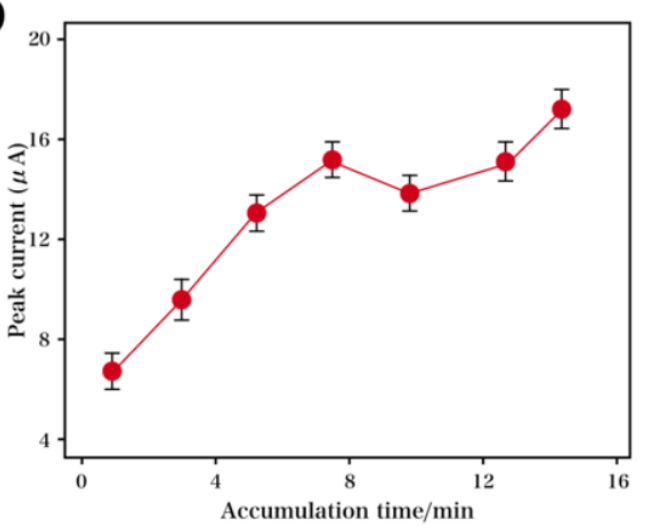

Figure 3. Effects of (A) pH value, (B) MWNT suspension volume, (C) accumulation potential and (D) accumulation time on the DPASV peak current of $0.1 \mathrm{M} \mathrm{Pb}^{2+}$

Fig. 3B shows the $1.5 \mathrm{mg} / \mathrm{mL}$ MWNT/GCE suspension volume effect on the stripping peak current. The increase in the MWNT/GCE suspension volume on the GCE surface generated a sharp increase in the stripping peak current of $\mathrm{Pb}^{2+}$, while the peak current was reduced with an excess of 
suspension volume, which may be due to an increase in resistance of the modified film against the mass transfer along with charge transfer for $\mathrm{Pb}^{2+}$ ions in the presence of excess suspension volume. Therefore, the optimal volume was determined as $5 \mu \mathrm{L}$.

The accumulation step is an effective and facile strategy for the enhanced voltammetric analysis sensitivity. Fig. 3C shows the accumulation potential effect on the stripping peak current of $\mathrm{Pb}^{2+}(-0.9 \mathrm{~V}$ to $-1.3 \mathrm{~V})$. When the accumulation potential reached $-1.1 \mathrm{~V}$, the peak current was optimal; thus, the following experiments used the accumulation potential of $-1.1 \mathrm{~V}$.

Fig. 3D shows the influence of the accumulation time on the stripping peak current of $\mathrm{Pb}^{2+}$ over a range of 2 to $14 \mathrm{~min}$. As the accumulation time increased to $10 \mathrm{~min}$, an increase in the peak current was found. Further increase caused a gradual decrease in the peak current. This can be attributed to the increase in thickness of the composite film as the accumulation time increased, which would affect the electron transfer rate of metal stripping [44, 45]. Therefore, the optimum accumulation time was determined as $10 \mathrm{~min}$ for the best sensitivity.

The potential interferents in the $\mathrm{Pb}^{2+}$ analysis process using the MWNT-coated GCE were selected from the main components of the test samples. To assess the selectivity performance of the MWNTs, DPASV experiments were carried out in a pH 5.0 acetate buffer solution in the presence of a $10 \mathrm{nM} \mathrm{Pb}^{2+}$ solution with varying amounts of metal ion interferents. The results obtained under the optimum parameters displayed that a 100-fold molar excess of $\mathrm{Hg}^{2+}, \mathrm{Cd}^{2+}, \mathrm{Cu}^{2+}$, and $\mathrm{Ag}^{+}$, and a 200fold molar excess of $\mathrm{Cr}^{3+}, \mathrm{Ni}^{2+}, \mathrm{Mn}^{2+}, \mathrm{Bi}^{3+}, \mathrm{Zn}^{2+}$, and $\mathrm{Sn}^{2+}$ showed no effect on the analysis of 10 $\mathrm{nM} \mathrm{Pb}{ }^{2+}$, and a relative error $<5 \%$ was obtained.

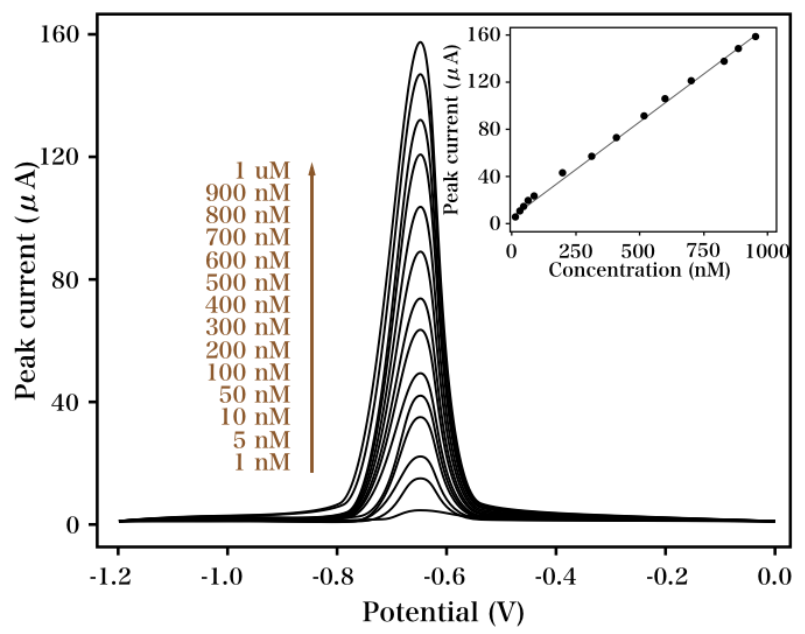

Figure 4. Stripping voltammograms of $\mathrm{Pb}^{2+}$ with varying concentrations at the MWNT-coated GCE (from bottom to top).

Fig. 4 shows the stripping voltammograms of the MWNT-modified GCE in the presence of varying concentrations of $\mathrm{Pb}^{2+}$. The detection analysis of our developed strategy toward $\mathrm{Pb}^{2+}$ was carried out under optimum parameters. For the calibration curve, the regression equation over a $\mathrm{Pb}^{2+}$ concentration range of $1 \mathrm{nM}$ to $1 \mu \mathrm{M}$ was as follows: $i_{\mathrm{p}}(\mu \mathrm{A})=1.5961 C(10 \mu \mathrm{M})+0.3715\left(R^{2}=0.99\right)$. 
However, the limit of detection (LOD) was $0.5 \mathrm{nM}(n=20)$ based on a signal-noise ratio of 3, showing that the developed sensor was highly sensitive to the analysis of lead in the water. The comparison of performance of our proposed electrode with those reported previously is presented in Table 1. Therefore, our proposed electrode could potentially be applied to the practical determination of $\mathrm{Pb}^{2+}$ due to the low LOD, high sensitivity, wide linear calibration range, and facile electrode preparation process.

Table 1. Behavior comparison of our developed MWNT-coated GCE and those proposed in other works.

\begin{tabular}{cccc}
\hline Electrode & LOD $(\mu \mathrm{M})$ & Linear range $(\mu \mathrm{M})$ & Reference \\
\hline PPR/GCE & 0.1 & $0.5-100$ & {$[46]$} \\
Thick-film carbon sensor & 0.0005 & $0.001-1$ & {$[43]$} \\
Cu based electrochemical sensor & 0.022 & $0.1-0.5$ & {$[47]$} \\
Bi(NO)/GCE & 0.00525 & $0.0075-12.5$ & {$[48]$} \\
NHAP/Nafion & 0.001 & $0.005-0.8$ & {$[49]$} \\
MWNTs coated GCE & 0.0005 & $0.001-1$ & This work \\
\hline
\end{tabular}

For the same electrode, 6 consecutive experiments were carried out for the detection of 10 $\mathrm{nM} \mathrm{Pb}^{2+}$ with an RSD of 4.26\%. Furthermore, $6 \mathrm{MWNT}$-coated GCEs were fabricated following the same procedure and were employed for the detection of $10 \mathrm{nM} \mathrm{Pb}^{2+}$ under optimal parameters with an RSD of $3.96 \%$. Therefore, our promoted electrode was highly reproducible and stable for the analysis of $\mathrm{Pb}^{2+}$.

Under laboratory conditions, the MWNT-coated GCE was used for the determination of $\mathrm{Pb}^{2+}$ in two environmental hot spring water samples collected from Aer Mountain, Inner Mongolia. The detection results of $\mathrm{Pb}^{2+}$ in real samples were displayed in Table 2. For the four real water samples, the as-prepared electrode showed the ability to distinctly detect $\mathrm{Pb}^{2+}$ and can be employed for the fabrication of green and portable sensors.

Table 2. Electrochemical performance of the MWNT-coated GCE toward the amount of $\mathrm{Pb}^{2+}$ in the hot spring water samples collected from Aer Mountain, Inner Mongolia.

\begin{tabular}{ccccc}
\hline Sample & Added $(\mathrm{nM})$ & Found $(\mathrm{nM})$ & Recovery $(\%)$ & RSD $(\%)$ \\
\hline Sample 1 & 100 & 99.71 & 99.71 & 4.51 \\
Sample 2 & 200 & 198.47 & 99.24 & 3.05 \\
Sample 3 & 300 & 311.41 & 103.80 & 2.99 \\
Sample 4 & 500 & 488.69 & 97.74 & 3.74 \\
\hline
\end{tabular}

\section{CONCLUSIONS}

In the present study, a fast and highly sensitive electrochemical strategy was developed for $\mathrm{Pb}^{2+}$ detection based on the synergistic properties of MWNTs and Nafion. MWNTs are characterized by 
high adsorption capacity, distinct catalytic activity, and high surface area; meanwhile, Nafion has a high cation exchange capacity. The developed electrode was further used for the detection of $\mathrm{Pb}^{2+}$ in real water samples.

\section{References}

1. S. Dong and Z. Sun, Journal of Applied Electrochemistry, 38 (2008) 1223.

2. V.B.D. Santos, E.L. Fava, N.S.D.M. Curi, R.C. Faria, T.B. Guerreiro and O. Fatibello-Filho, Analytical Methods, 7 (2015) 3105.

3. C. Kokkinos, A. Economou, N.G. Goddard, P.R. Fielden and S.J. Baldock, Talanta, 153 (2016) 170.

4. X. Wei, Z. Lan and S. Liu, Chem. Eng. J., 284 (2016) 650.

5. M. Li, J. Wu, L. Cui and H. Ju, Journal of Electroanalytical Chemistry, 757 (2015) 23.

6. T. Alemayehu, K. Siraj, B. Desalegn Assresahegn and T. Entele Tesema, Advanced Electrochemistry, 1 (2013) 48.

7. I. Šestáková, D. Miholová, A. Slámová, P. Mader and J. Száková, Electroanalysis, 6 (2010) 1057.

8. F. Arduini, J.Q. Calvo, G. Palleschi, D. Moscone and A. Amine, Trac Trends in Analytical Chemistry, 29 (2010) 1295.

9. J. Huang, S. Bai, G. Yue, W. Cheng and L. Wang, Rsc Advances, 7 (2017) 28556.

10. S. Morante-Zarcero, D. Pérez-Quintanilla and I. Sierra, Journal of Solid State Electrochemistry, 19 (2015) 2117.

11. T.P. Ferreira, R.A.D. Sousa and D. Lowinsohn, Analytical Methods, 8 (2016) 8028.

12. T.F. Tormin, G.K.F. Oliveira, E.M. Richter and R.A.A. Munoz, Electroanalysis, 28 (2016) 940.

13. A. Bala, M. Pietrzak, Ł. Górski and E. Malinowska, Electrochimica Acta, 180 (2015) 763.

14. H. Xing, J. Xu, X. Zhu, X. Duan, L. Lu, Y. Zuo, Y. Zhang and W. Wang, Journal of Electroanalytical Chemistry, 782 (2016) 250.

15. M.P. Bui, C.A. Li, K.N. Han, X.H. Pham and G.H. Seong, Analytical Sciences, 28 (2015) 699.

16. N. Serrano, A. Gonzálezcalabuig and V.M. Del, Talanta, 138 (2015) 130.

17. J. Singh, H. Singh, T. Pandiyan and N. Singh, Electroanalysis, 27 (2016) 2544.

18. D. Wang, Y. Ke, D. Guo, H. Guo, J. Chen and W. Weng, Sensors \& Actuators B Chemical, 216 (2015) 504.

19. A. Afkhami, F. Kafrashi and T. Madrakian, Ionics, 21 (2015) 2937.

20. Y. Wang, K. Du, Y. Chen, Y. Li and X. He, Analytical Methods, 8 (2016) 3263.

21. J. Wang, X. Chen, K. Wu, M. Zhang and W. Huang, Electroanalysis, 28 (2016) 63.

22. W. Kang, X. Pei, C.A. Rusinek, A. Bange, E.N. Haynes, W.R. Heineman and I. Papautsky, Anal. Chem., 89 (2017) 3345.

23. W. Zhou, C. Li, C. Sun and X. Yang, Food Chemistry, 192 (2016) 351.

24. F. Zhan, F. Gao, X. Wang, L. Xie, F. Gao and Q. Wang, Microchim. Acta., 183 (2016) 1169.

25. S. Mu, Y. Liang, S. Chen, L. Zhang and T. Liu, Materials Science \& Engineering C Materials for Biological Applications, 50 (2015) 294.

26. H. Zhang, Y. Yuan, F. Yang, N. Zhang and X. Cao, Rsc Advances, 5 (2015) 38630.

27. D. Adolphe, D. Adolphe, D. Adolphe, G.L. Potop and M. Agop, Journal of Nanomaterials, 2015 (2015) 6.

28. M. Rashid, T.S. Jun, Y. Jung and S.K. Yong, Sensors \& Actuators B Chemical, 208 (2015) 7.

29. G. Ziyatdinova, K. Os'Kina, E. Ziganshina and H. Budnikov, Analytical Methods, 7 (2015) 8344.

30. Y.J. Yang and W. Li, Russian Journal of Electrochemistry, 51 (2015) 218.

31. S.Y. And and S. Michielsen, Chemical Engineering Science, 35 (2017) 10108.

32. R. Li, D. Guo, J. Ye and M. Zhang, The Analyst, 140 (2015) 3746.

33. H. Wei, X.S. Wu, G.Y. Wen and Y. Qiao, Molecules, 21 (2016) 617. 
34. Y.J. Yang and W. Li, Ionics, 21 (2015) 1121.

35. S. Cerovac, V. Guzsvány, Z. Kónya, A.M. Ashrafi, Š. I, S. Rončević, Á. Kukovecz, B. Dalmacija and K. Vytřas, Talanta, 134 (2015) 640.

36. F. Fasihi, F. Farjami and G.H. Shafiee, Rsc Advances, 5 (2015) 95087.

37. H. Wang, Y. Wu and J.F. Song, Biosensors \& bioelectronics, 72 (2015) 225.

38. Y. Wang, H. Ge, Y. Wu, G. Ye, H. Chen and X. Hu, Talanta, 129 (2014) 100.

39. M. Salarian, A. Ghanbarpour, M. Behbahani, S. Bagheri and A. Bagheri, Microchim. Acta., 181 (2014) 999.

40. S. Chaiyo, E. Mehmeti, Ž. K, W. Siangproh, O. Chailapakul and K. Kalcher, Anal. Chim. Acta., 918 (2016) 26.

41. S. Anandhakumar and J. Mathiyarasu, Microchim. Acta., 180 (2013) 1065.

42. X. Dai, O. Nekrassova, M.E. Hyde and R.G. Compton, Analytical chemistry, 76 (2004) 5924.

43. G.S. Reeder and W.R. Heineman, Sensors and Actuators B: Chemical, 52 (1998) 58.

44. H. Li, J. Li, Z. Yang, Q. Xu, C. Hou, J. Peng and X. Hu, J. Hazard. Mater., 191 (2011) 26.

45. J. Wang, Ü.A. Kirgöz and J. Lu, Electrochemistry Communications, 3 (2001) 703.

46. G. Yang, X. Qu, M. Shen, C. Wang, Q. Qu and X. Hu, Microchim. Acta., 160 (2008) 275.

47. W. Kang, P. Xing, C.A. Rusinek, A. Bange, E.N. Haynes, W.R. Heineman and I. Papautsky, Anal. Chem., 89 (2017) 3345.

48. L.T. Tufa, K. Siraj and T.R. Soreta, Russian Journal of Electrochemistry, 49 (2013) 59.

49. D.W. Pan, Y.N. Wang, Z.P. Chen, T. Lou and Q. Wei, Anal. Chem., 81 (2009) 5088

(C) 2018 The Authors. Published by ESG (www.electrochemsci.org). This article is an open access article distributed under the terms and conditions of the Creative Commons Attribution license (http://creativecommons.org/licenses/by/4.0/). 